\title{
The impact of detoxification on the coping strategies in a sample of inpatient alcoholics
}

\author{
Elias Tzavellas*, Thomas Paparrigopoulos, Dimitrios Karaiskos, \\ Nikos Stefanis, Agellos Komporozos and John Liappas
}

Address: Department of Psychiatry, Eginition Hospital, Athens University Medical School, Athens, Greece

* Corresponding author

from International Society on Brain and Behaviour: 3rd International Congress on Brain and Behaviour

Thessaloniki, Greece. 28 November - 2 December 2007

Published: 17 April 2008

Annals of General Psychiatry 2008, 7(SuppI I):S325 doi:I0.I I86/I744-859X-7-SI-S325

This abstract is available from: http://www.annals-general-psychiatry.com/content/7/SI/S325

(C) 2008 Tzavellas et al.; licensee BioMed Central Ltd.

\section{Background}

In our study we investigate some aspects of the coping strategies and the impact of detoxification on these characteristics in a sample of inpatient alcohol dependent individuals.

\section{Materials and methods}

The sample of the study comprised 200 Caucasian, Greek nationality patients (147 males, 53 females) randomly selected over a 3-year period, who fulfilled the DSM-IV diagnostic criteria for alcohol abuse/dependence, treated on an inpatient basis at the specialized drug and alcohol addiction service of the Athens University Psychiatric Clinic at the Eginition Hospital. Detoxification treatment was comprised of vitamin replacement (vitamin B complex, vitamin $\mathrm{C}$, vitamin $\mathrm{E}$ ) and oral administration of diazepam (30-60 mg daily in divided doses), with a gradual taper off over a week. Subjects were assessed with the Pilowski scale for hypochondria, the Leyton scale for obsessive-compulsive symptoms and the Sifneos scale for alexithymia. The questionnaires were administered at the beginning of the detoxification period and at discharge. Descriptive statistics are used for the presentation of results.

\section{Results}

Mean age \pm SD of the sample was $47,4 \pm 11,7$ years and mean daily alcohol consumption was $359,9 \pm 266,8 \mathrm{gr} /$ day. Upon admission the mean scores on the different scales were as follows: Leyton: $14,8 \pm 2,7$, Pilowski: $9,32 \pm$
2,63, Sifneos: $11,3 \pm 2,6$. After completion of detoxification the scores were: Leyton: 9,07 $\pm 3,05 \quad(\mathrm{P}<.000)$, Pilowski: 4,67 $\pm 2,58(\mathrm{p}<.000)$, and Sifneos: $10,6 \pm 2,2$ (NS).

\section{Conclusions}

Detoxification from alcohol appears to significantly change some aspects of the coping strategies of alcohol dependent individuals. Further investigation of this issue should be undertaken. 